\title{
In-Space Manufacturing at NASA Marshall Space Flight Center: A Portfolio of Fabrication and Recycling Technology Development for the International Space Station
}

\author{
Tracie Prater ${ }^{1}$, Niki Werkheiser ${ }^{2}$, Frank Ledbetter ${ }^{3}$, and Kristin Morgan ${ }^{4}$ \\ NASA Marshall Space Flight Center, Huntsville, AL, USA
}

The in-space manufacturing project at NASA Marshall Space Flight Center seeks to mature the manufacturing capabilities which will be needed on long duration, long endurance human spaceflight missions. The ability to manufacture materials and parts in space rather than launching them from earth has the potential to reduce logistics requirements and enhance crew safety. The International Space Station serves as a unique orbiting test bed for in-space manufacturing technology development for NASA and its commercial partners. This paper provides an overview of the projects currently in the in-space manufacturing technology portfolio and key technology development efforts in the past year.

\section{Introduction}

Since its inception in 2014, NASA's in-space manufacturing (ISM) project has partnered with industry to develop the technologies needed to provide an on-demand manufacturing capability for the International Space Station (ISS) and exploration-class missions beyond ISS. Logistics analyses strongly indicate that future crewed missions where cargo resupply is limited pose significant supportability challenges unless a robust manufacturing capability is available to support sparing, reduce the amount of material which must be upmassed, and enhance crew safety by providing an option for quick response manufacturing that enables rapid adaptation to unforeseen system issues. ${ }^{1,2}$ These analyses also show ISM can reduce uncertainty in sparing and impart an additional level of redundancy. The impact of ISM from a mission planning perspective is maximized when complemented by material recycling and in situ resource utilization (ISRU) technologies to produce feedstocks using mission recyclables and indigenous materials. ISM is seen as a key enabler for sustainable and safe travel beyond low earth orbit.

\section{3D Printing in Zero G Technology Demonstration Mission: Phase II Results}

The in-space manufacturing project began in 2014 with the first 3D printer developed for the International Space Station. This hardware, built by Made in Space, Inc. under a small business innovative research (SBIR) contract, was part of the 3D Printing in Zero G technology demonstration mission. The printer uses the fused filament fabrication (FFF) process, where a continuous filament of thermoplastic is fed through a heated extruder and deposited on a build tray layer by layer. The extruder head and/or bed are moved in a controlled manner to deposit the filament in the desired shape. The printer from the technology demonstration mission uses ABS filament and to date has completed two rounds of operations on-orbit, printing a total of 55 parts. Phase I operations took place from November-December 2014. The results of analysis of these specimens and comparison with ground-based specimens made with the printer prior to its launch to ISS are summarized in reference 3 and 4. Some differences were noted between the flight specimens and analogous ground specimens, but these were hypothesized to be a result of process variability rather than the influence of microgravity on the FFF process. Phase II operations, conducted in June and July 2016, allowed for an additional investigation of microgravity effects on FFF and produced a

\footnotetext{
${ }^{1}$ Aerospace engineer, Materials and Processes Laboratory, NASA MSFC, AIAA senior member.

${ }^{2}$ In-Space Manufacturing project manager, Science and Technology Office, NASA MSFC

${ }^{3}$ Senior technical adviser, MIPSS SME contract, NASA MSFC

${ }^{4}$ Science and Technology Office, NASA MSFC
} 
total of 34 prints. These specimens underwent extensive evaluation at NASA Marshall Space Flight Center throughout 2016 and 2017. Comprehensive results of the phase I and phase II specimen analysis were published as a NASA technical report ${ }^{5}$. A holistic look at the phase I and phase II data detected no significant differences in mass, density, dimensions, or mechanical strengths of geometrically similar specimens, regardless of manufacturing setting (specifically the distance between the extruder and the build tray, which was varied for phase I prints) or manufacturing environment (ISS or ground-manufactured). No evidence of a substantive microgravity effect on material structure was observed with scanning electron microscopy and computed tomography (CT). Additionally, chemical analysis of the filament feedstock from phase II using Fourier Transform Infrared Spectroscopy (FTIR) showed no significant changes in chemical composition as a result of aging (phase II feedstock was 18 months older than phase I feedstock at the time of printing). Phase II data strongly indicated that differences between phase I ground and flight specimens were attributable to build to build variability and changes in manufacturing process settings onorbit rather than operation of the FFF process and/or the technology demonstration hardware in a microgravity environment. The lack of an engineering significant effect of microgravity on material outcomes suggests that specimens produced with a ground-equivalent printer should be representative of material produced on-orbit with the FFF process.

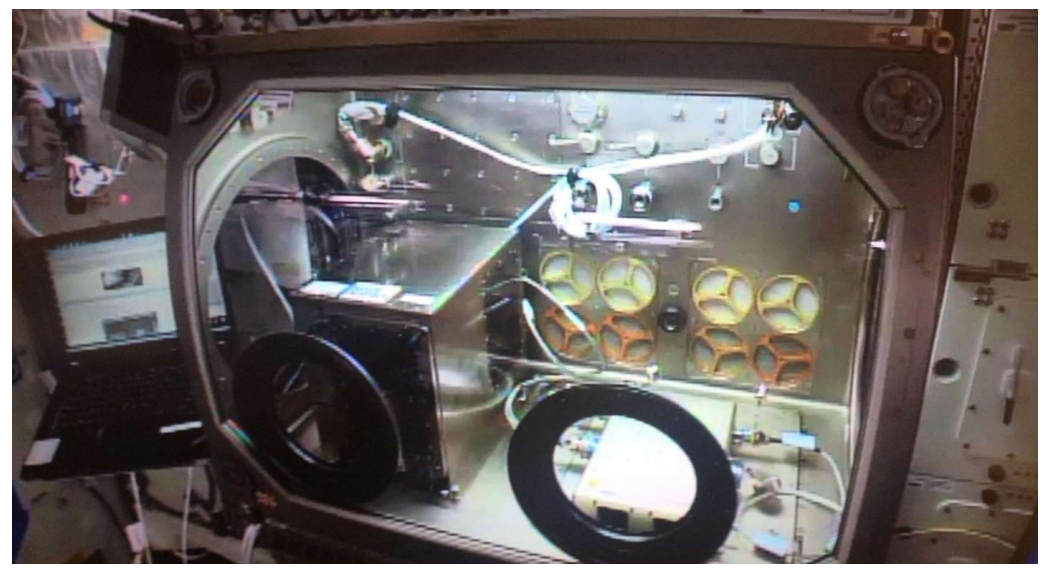

Figure 1. 3D Printing in Zero G Technology Demonstration printer operating in the Microgravity Science Glovebox (MSG).

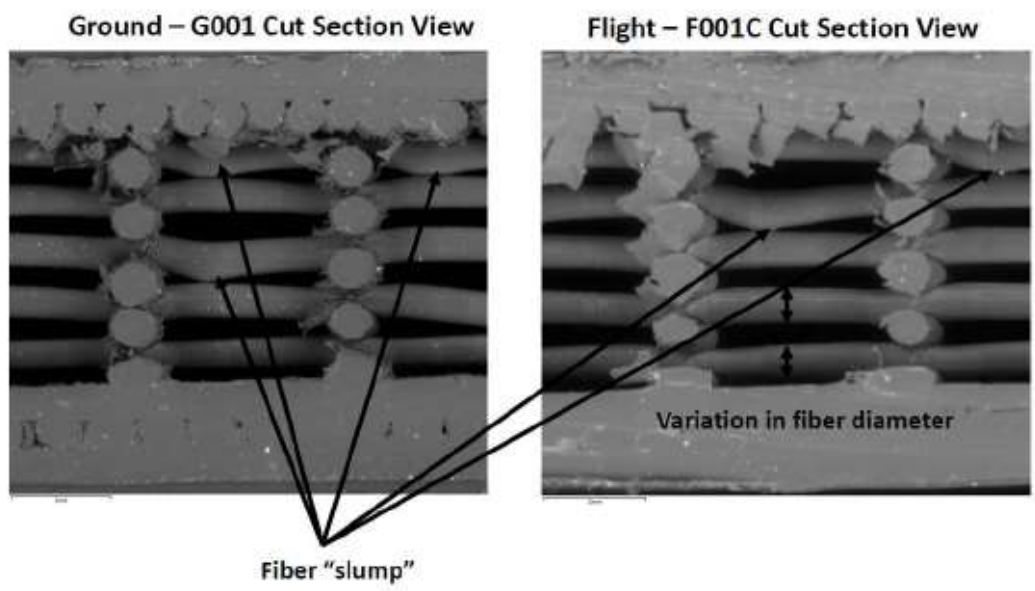

Figure 2. Examples of scanning electron microscope (SEM) images of specimens from the 3D Printing in Zero G Technology Demonstration Mission. 


\section{Additive Manufacturing Facility Materials Characterization}

The 3D printing in zero $\mathrm{G}$ technology demonstration mission proved the feasibility of operating an FFF system in microgravity. Following this mission, Made in Space developed the Additive Manufacturing Facility (AMF), a commercial, multimaterial 3D printing facility on ISS that NASA is one user for. AMF can print Acrylonitrile Butadiene Styrene (ABS), High Density Polyethylene (HDPE), and polyetherimide $(\mathrm{PEI})^{6}$. NASA is currently testing ISS-manufactured specimens from AMF as part of an extensive characterization plan to baseline design properties for this printer. This work includes evaluation of groundmanufactured specimens produced with a flight equivalent printer and offers an additional opportunity to evaluate the existence of a substantive link between microgravity and material outcomes. While it is unlikely that microgravity influences FFF based on analysis of phase I and phase II specimens from the technology demonstration discussed previously, differences in manufacturing process variables between ground and flight specimens added complexities to the assessment.

Table 1 identifies the AMF test matrix, to be applied to both ground and on-orbit printed materials. Identical manufacturing process parameters were used for the ground-equivalent and flight printers. Sixty mechanical test specimens (listed in Table 1) were machined from panels produced by both the ground and flight printer, for a total of 120 mechanical specimens. The testing methodology for this work is similar to composites; the test coupons are not printed directly but extracted from 0.25 " thick panels of 3D printed material. The test matrix also evaluates the impact of orientation on material properties. The throughthickness strength of the material, which is the bounding condition for lowerbound strength, is evaluated using the flatwise tension test per ASTM C2977. In addition to the mechanical test specimens, two range coupons will be produced on both the ground and flight printer. These specimens will enable evaluation of printer performance: resolution, dimensional accuracy, and tolerances. One functional part, the EMU fan cap, will be produced on both the ground and flight printer. This part will be fabricated in two pieces and provide an evaluation of fit-up at interfaces.

Table 1. Additive Manufacturing Facility Materials Characterization Test Matrix.

\begin{tabular}{|l|l|l|l|}
\hline $\begin{array}{l}\text { Specimen Type, } \\
\text { Orientation }\end{array}$ & Quantity (ground) & Quantity (flight) & ASTM standard \\
\hline Tension, 0 & 10 & 10 & D638 \\
\hline Tension, 90 & 10 & 10 & D638 \\
\hline Compression, 0 & 10 & 10 & D695 \\
\hline Compression, 90 & 10 & 10 & D695 \\
\hline Tension, +/-45 (shear) & 10 & 10 & D3518 \\
\hline Flatwise tension & 10 & 10 & C297 \\
\hline Range coupon & 2 & 2 & n/a \\
\hline EMU fan cap & 1 & 1 & n/a \\
\hline
\end{tabular}

Currently AMF serves as the utilization printer for ISS. To date, NASA has printed multiple functional parts for ISS using AMF: an oxygen generation system adapter to fixture a flow meter that measures the health of Environmental Control and Life Support Systems (ECLSS) hardware on ISS, the multifunctional crew tool which won the Future Engineers student design challenge, a tow hitch for the SPHERES payload, a cover for a radiation sensor in the BEAM module, and a small antenna feed horn (Figure 3) ${ }^{8}$. Development of baseline design values through the plan previously outlined above will support future on-orbit, ondemand fabrication of both original and replacement components of ABS with AMF. Additional test plans may be expanded to include PEI or HDPE, based on NASA material use cases. At the time of this writing, ground-manufactured AMF specimens are undergoing testing and evaluation at Southern Research Institute 
in Birmingham, Alabama. Half of the flight specimens have been printed and are currently in testing and evaluation. The remainder of the specimens are set to be printed on ISS in mid-August.
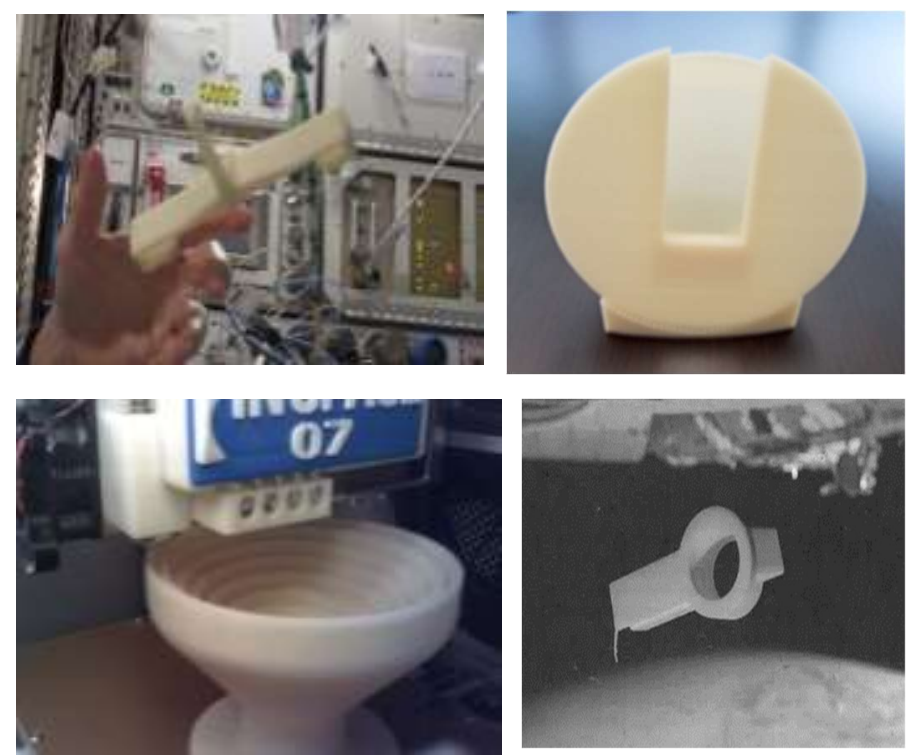

Figure 3. Examples of parts printed on AMF: SPHERES Tow Hitch (upper left), BEAM module radiation sensor cover (upper right), antenna feedhorn (lower left), oxygen generation system adapter (lower right).

\section{Tethers Unlimited ReFabricator Payload}

The Refabricator payload was developed by the company Tethers Unlimited under a small business innovative research (SBIR) contract and will be operational on ISS in 2018. ReFabricator is an integrated 3D printer and recycler which repurposes printed Ultem 9085 (PEI/PC) into high-quality 3D printer filament ${ }^{9}$. Once the payload is installed in an EXPRESS rack onboard the ISS, the unit will complete seven printing/recycling cycles. Each printing cycle will produce tensile specimens (for downmass) and a block to serve as the input material for the recycler. Each recycling cycle will produce filament for further printing; some filament from each cycle will remain on the spool for further analysis. Following phase I payload operations, materials specimens will be returned to earth for testing and evaluation, including mechanical testing and chemical analysis.

The ReFabricator represents the first repeatable, closed-loop process for recycling plastics in the microgravity environment. The system mitigates contamination of scrap material, minimizes damage to the material during reprocessing, and controls the melting and resolidification of the material to ensure best material outcomes. As noted in ISM logistics analyses, recycling is needed for long duration missions to minimize the use of external resources. The ReFabricator also enables use of polymers which would otherwise represent nuisance/trash materials on a space mission. When combined with in situ resource utilization technologies, recycling is a powerful tool for reducing the initial mass requirements for space missions. The ReFabricator will serve as the first proof of concept for recycling in space, providing a key assesssment of the effects of microgravity on the recycling process and the consistency of materials produced over multiple cycles on-orbit. Knowledge gained from the payload will advance the state of the art for 3D printing/recycling technologies on earth and inform design of future recycling systems for exploration missions where resources are limited. 


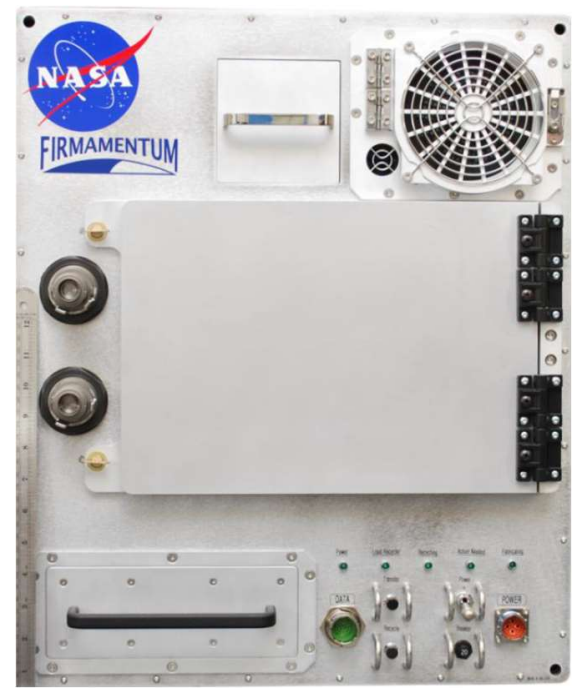

Figure 4. The ReFabricator Engineering Test Unit.

\section{Other Small Business Innovative Research (SBIR) activities}

In-space manufacturing continues to leverage the small business innovative research (SBIR) program to develop payloads, conduct materials research, and assess the feasibility of candidate manufacturing processes for evolution to a space-based capability. Phase I efforts (maximum of $\$ 125,000$ ) are intended to establish the technical feasibility of a project, its scientific merit, and its commercial potential. Phase II efforts (maximum of $\$ 750,000$ ) focus on further development of the innovation investigated under phase I. Phase III is commercialization and development of a product (hardware for ISM payloads currently on ISS were developed through phase III SBIRs).

In 2017 and 2018, the in-space manufacturing project has overseen the development efforts listed below. This work spans the areas of hybrid manufacturing and development of metallic 3D printing capabilities for ISS, development of common use/recyclable materials, sterilization of recycled materials, and printable electronics.

- Phase II SBIR: Vulcan Advanced Hybrid Manufacturing System from Made in Space. Phase I concluded in December 2017. This project focuses on development of a multimaterial, hybrid manufacturing capability for ISS. The system includes fused filament fabrication (FFF), a weldbased additive manufacturing process for metal fabrication, a CNC mill for processing, and an automated capability for movement of the part between subsystems ${ }^{11}$. Phase I consisted of extensive trade studies for the system and its manufacturing processes/materials, initial material evaluations, and demonstrations of constituent subsystems, including a chip capture system for debris generated during the manufacturing process. Phase II work includes design, construction, and testing of an integrated, prototype unit to perform hybrid manufacturing functions. A metal part produced with the Vulcan capability is shown in Figure 5. 


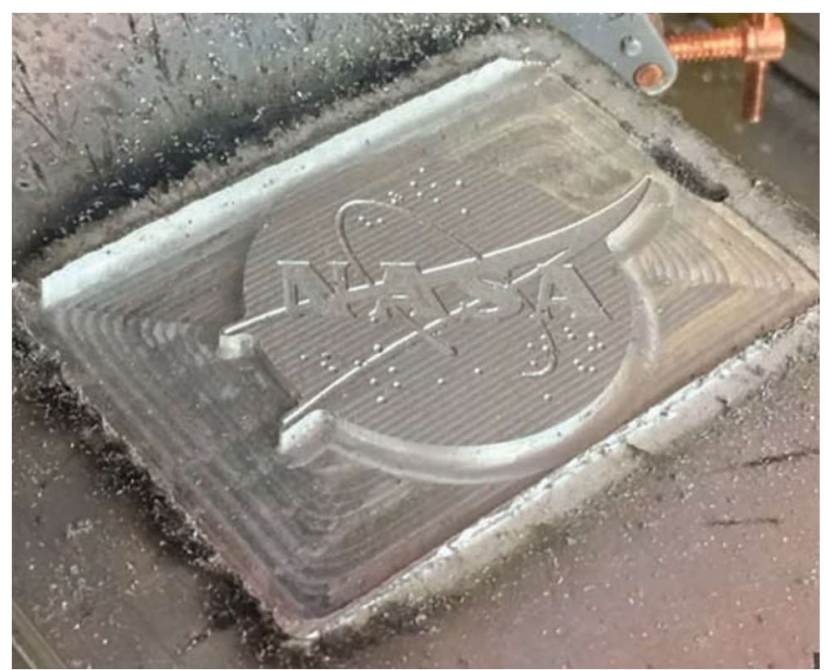

Figure 5. Part produced with Vulcan system. Image courtesy of Made in Space, Inc.

- Phase II SBIR: ISS Fabrication Laboratory using Ultrasonic Additive Manufacturing Technology from Ultra Tech Machinery. Phase I concluded in December 2017. In phase I, this project evaluated the feasibility of scaling the ultrasonic additive manufacturing process to fit within the mass, power, and volume constraints of ISS. A solid-state process that occurs at room temperature, ultrasonic additive manufacturing (UAM) uses sound waves to remove the oxide layer between adjacent layers of metal foil, creating a solid state bond (Figure 6). UAM has a long history in Aluminum alloys and multimaterial systems which may be difficult to manufacture using conventional processes (ex. Metal Matrix Composites, Functionally Graded Materials $)^{12}$. In the phase I effort, Ultra Tech and subcontractor Fabrisonic, Inc. designed and tested a $30 \mathrm{kHz}$ sonotrode which reduced process forces to enable design of a system compatible with ISS requirements. Phase I also included design of a motion system for the integrated development unit (which includes a CNC), FEA analysis to size components for the eventual system, and a study of the characteristic size of chip debris. Early tests of the prototype system demonstrated quality welds in 6016 T6 and 7075 T6 and enabled material production at significantly lower power and forces. Development of the phase II system, currently underway, includes the addition of a CNC mill head to enable finishing of parts. UAM has previously been used by NASA to print heat exchangers, embed sensors in metal components, and produce metal matrix composites.

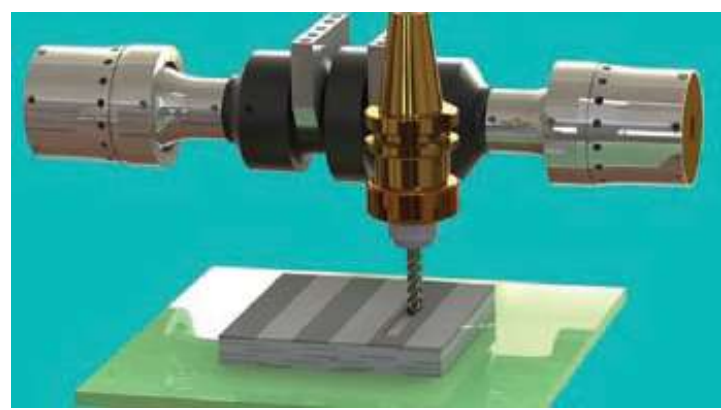

Figure 6. Illustration of the Ultrasonic Additive Manufacturing process. Image courtesy of Ultra Tech. 
- Phase II SBIR: Metal Advanced Manufacturing Bot-Assisted Assembly (MAMBA) Process from Tethers Unlimited. Phase I effort concluded in December 2017. MAMBA (Figure 7) combines three technologies to provide a precision metallics manufacturing capability for ISS: a press that processes virgin or scrap material into a metal ingot, a CNC mill designed to operate in microgravity, and a robotic assistant to facilitate automated processing of material/parts through the subsystems ${ }^{13}$. MAMBA applies the same Positrusion process used to recycle plastics in the ReFabricator to aerospace grade metals. Phase II work will focus on development of the integrated prototype system.

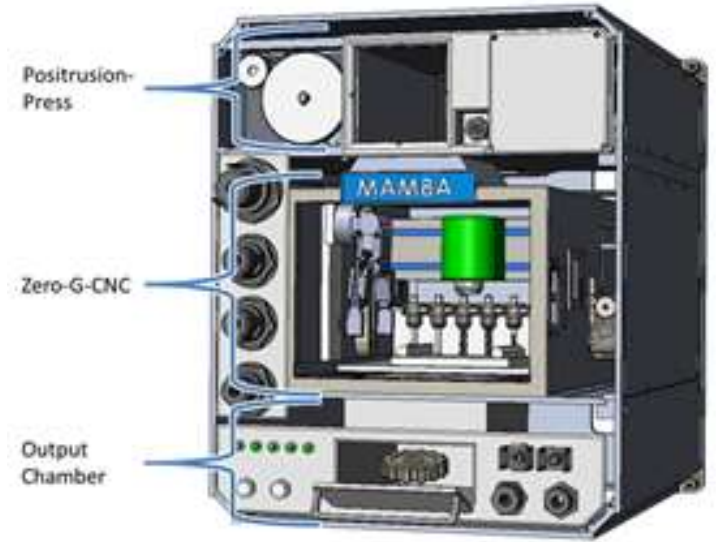

Figure 7. TUI's MAMBA system consists of the Positrusion press, a zero-G CNC, and an output chamber. Parts are processed through the system using a robotic arm. Image courtesy of Tethers Unlimited.

- Phase II SBIR: ERASMUS from Tethers Unlimited. Medical-Grade and Food-Safe Plastic Recycling and Sanitization System. ERASMUS will enable use of recycled materials for medicalgrade and food-safe applications on ISS ${ }^{14}$. Since bacteria and viruses are more virulent in space and crew immune systems tend to be compromised, sterility of devices produced using recycled materials is critical. ERASMUS provides a dry heat sterilization process and UV sanitization routine which can be integrated with $3 \mathrm{D}$ printing and recycling systems. Figure 8 shows examples of biomedical and food tools manufactured with ERASMUS to date.

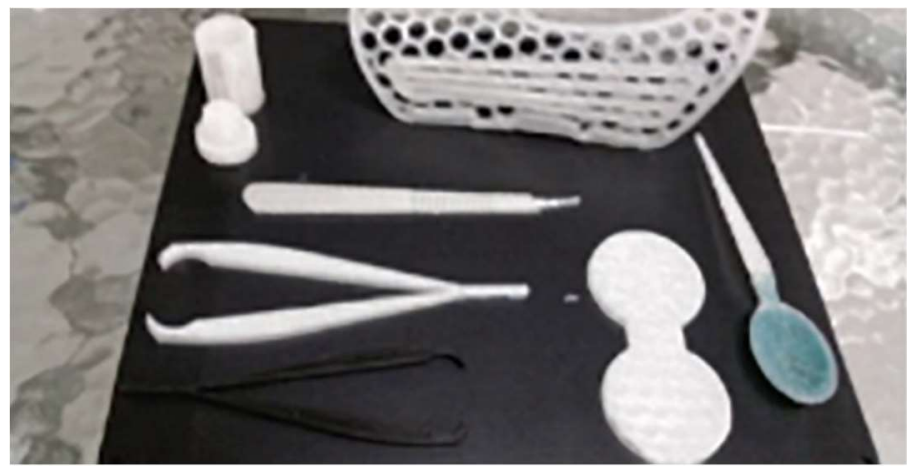

Figure 8. Examples of biomedical and food tools manufactured with ERASMUS. Image courtesy of Tethers Unlimited. 
- Phase II SBIR: Sintered Inductive Metal Printer with Laser Exposure (SIMPLE) from Techshot. Techshot is developing a 3D metal printer in which a ferromagnetic wire metal filament is heated to its Curie temperature through induction and deposited on a build platform where a low power laser completes the melt ${ }^{15}$. The prototype unit is shown in Figure 9.

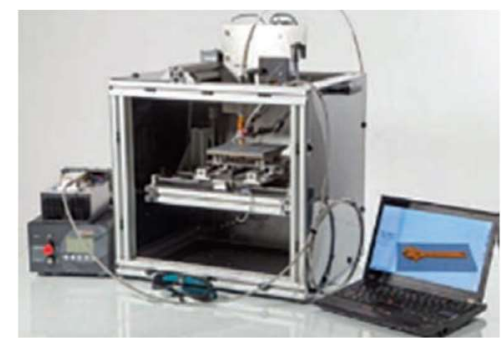

Figure 9. SIMPLE, a small wire-fed metal 3D printer from Techshot. Image courtesy of Techshot.

- Phase II-X SBIR: Customizable Recyclable International Space Station Packaging (CRISSP) from Tethers Unlimited. The CRISSP Phase II effort matured recyclable launch packaging materials to enable sustainable manufacturing and reuse of otherwise nuisance materials on deep space missions ${ }^{16}$. A process for 3D printing of customized foam packaging was developed and testing demonstrated that this packaging can provide vibration protection equivalent to or exceeding current materials. Test samples were recycled into filament for 3D printing and degradation studies assessed reduction in material characteristics over multiple recycling cycles. The CRISSP Phase II-X effort focuses on redesign and upgrade of TUI's ReFabricator system for multimaterial capabilities. This effort will increase the number of materials that can be recycled into feedstock filament and re-printed with the system. Degradation studies of materials in the recycling process will be assisted by Cornerstone Research Group (CRG).

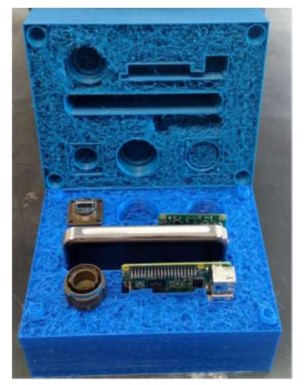

Figure 10. CRISSP foam packaging from Tethers Unlimited.

- Phase II-X SBIR: Reversible Copolymer Materials for FDM 3-D Printing of Non-Standard Plastics from Cornerstone Research Group (CRG). Under a phase II SBIR, CRG developed thermally-reversible polymer materials compatible with fused filament fabrication (FFF) 3Dprinting systems ${ }^{17}$. These materials are designed to be recycled, blended, and extruded. Additives can also be combined with existing waste packaging, enabling reclamation of filament for additive manufacturing from packaging materials. In the phase II-X effort, CRG will conduct further characterization of their thermally reversible material and scale the associated polymer resin production and packaging production processes. CRG will also perform the testing needed to certify this material for spaceflight applications 
- Phase I SBIR: STEPS Software and Tools for Electronics Printing in Space (STEPS) from Techshot. Phase I concluded in December 2017. STEPS was intended to complement NASA Marshall's in-house work on printable electronics and ink development by evolving a flightproven capability for manufacturing electronics via direct write printing. Historically, many ISS system failures are electronic in nature and the ability to repair or fabricate electronics would be a highly desirable capability for a crewed space mission. ${ }^{18}$

- Phase I SBIR: Adaptive laser sintering for in-space printed electronics from Optomec. Phase I concluded in 2017. The goal of the phase I project was to enhance the Optomec Aerosol Jet(R) technology for additive manufacturing of electronics through the addition of an Adaptive Laser Sintering System (ALSS) module. This enables a fully automated, end to end system for printed electronics and extends Optomec's Aeresol Jet technology to the printed electronics market ${ }^{19}$. ALSS can significantly reduce thermal damage to low Tg polymer substrates (such as acrylic, PET, etc.). during sintering of the metal inks, which is a current issue in additive electronics manufacturing.

Recent phase I SBIR awards in 2018 were in the area of development of high strength filament feedstock for in-space manufacturing and in-process inspection techniques. The ability to fabricate higher strength parts is essential to expand the use scenarios for in-space fused filament fabrication (FFF) capabilities. This requires development of novel materials and post-processing techniques for improving isotropy and reducing internal porosity (yielding parts with properties more comparable to injection-molded materials). Funded projects under this opportunity seek to significantly enhance the properties achievable with FFF techniques and expand the use of FFF processes for manufacturing (both in-space and on-earth) beyond low-criticality applications.

The ability to ensure production of parts with repeatable quality is also critical for ISM implementation. There are essentially two approaches to ensure consistency in parts and the manufacturing process: a traditional qualification and certification (which may be difficult for ISM due to constraints on crew time and equipment size limitations) and online quality control (i.e. process monitoring, where in situ monitoring of process signals provides information about the quality of a part produced by a manufacturing process). Qualification and certification processes for ISM will require better machine and feedback control than is currently available with off the shelf printers. While traditional approaches to qualification and certification are also being pursued, a more immediate solution is online/off-line quality control techniques that are uniquely adaptable to ISM. Three projects on real-time, in situ quality assurance of AM parts manufactured in the space environment were selected under this SBIR phase I project call in 2018.

\section{Multimaterial Fabrication Laboratory for the International Space Station}

Capabilities discussed thus far are intended to be evolutionary. The best materials, practices, and processes developed under the umbrella of in-space manufacturing will ultimately lead to a robust, integrated suite of manufacturing capabilities which can be deployed on exploration class missions postISS. In 2017, the in-space manufacturing project issued a broad agency announcement for the Multimaterial Fabrication Laboratory for the International Space Station ${ }^{20}$. The "FabLab" is intended to be a complex, integrated manufacturing solution capable of manufacturing precision parts for sparing, repair, and logistics support. FabLab in its flight incarnation will also have a remote commanding capability and a high degree of autonomy, as crew time is severely constrained for ISS operations due to the high volume of payloads (in addition, continuous tending of a manufacturing system by a crew member on a long duration mission represents an additional risk in itself). Development of inspection and diagnostic capabilities are also part of the FabLab effort. Current ISS systems are limited in their diagnostic and inspection capabilities and primarily rely on downmassing parts and materials to earth for ground-based evaluation. In situ techniques 
are under development for ground-based manufacturing systems that can also be leveraged by ISM. The minimum capabilities for the FabLab, as set forth in the broad agency announcement are outlined below:

- On-demand manufacturing of metallics and other materials in the microgravity environment

- Includes safety, waste management, and containment of debris

- Ability to process a range of metals for in-space applications

- Ability to operate in a reduced gravity environment

- Minimum build envelope of 6"x6"x6"

- Internal or external build envelope that is as large as possible

- High geometric part complexity and accuracy

- Ability to fit within EXPRESS rack constraints (ex. power, mass, volume)

- Earth-based remote commanding

- Remote commanding for all nominal tasks, including part removal and handling

- Post-processing requirement on crew for part readiness should be minimized

- In-line remote/autonomous inspection and quality control

- Incorporate inspection/verification capabilities to ensure quality control (assess tolerances, voids, etc.)

- Metallurgical quality of finished part

\section{Express Rack}

Lockers (8)

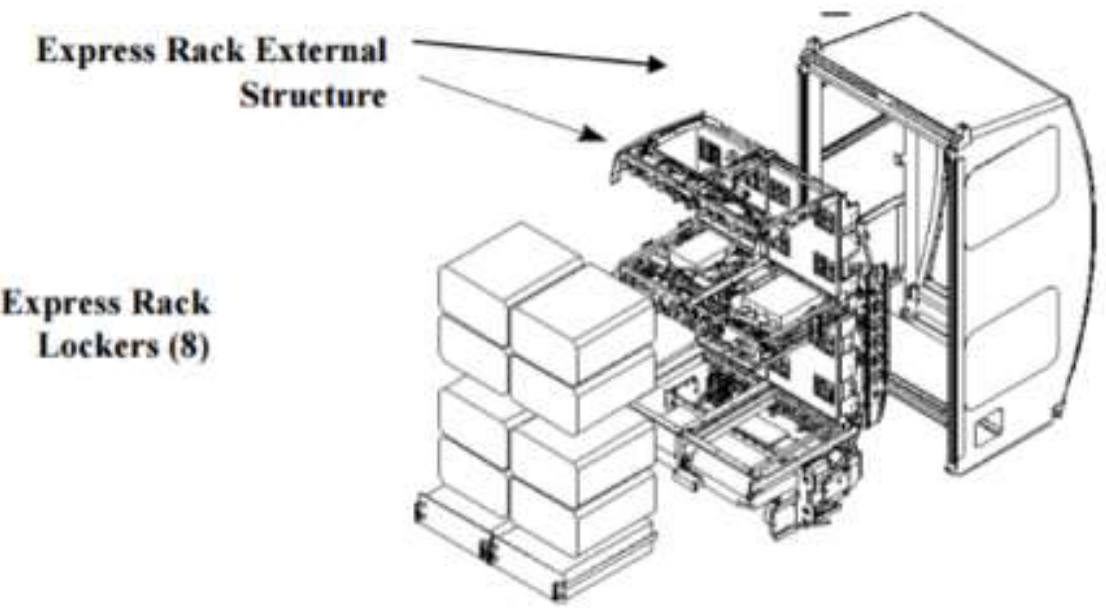

Figure 11. EXPRESS rack structure. Power consumption for FabLab is limited to $2000 \mathrm{~W}$, payload mass limit is $576 \mathrm{lbm}$.

The request for proposals for this opportunity closed in mid-September 2017 with award announcement in December 2017. FabLab is part of NASA's NEXT-STEP program and is intended to follow a phased development approach. Phase A awards are for an 18 month period of performance and culminate with development of a ground-based proof of concept with a design path towards system maturation. Over future funding efforts, FabLab will mature into a flight demonstration onboard the International Space Station in phase C. Companies working under phase A are: Interlog Corporation of Anaheim, California; Techshot, Inc. of Greeneville, Indiana; and Tethers Unlimited, Inc. of Bothell, Washington ${ }^{21}$. The total funding for the three awards under phase A (18 month effort) is 10.2 million dollars. Companies who did not participate in phase A are eligible to participate in subsequent phases of the Fabrication Laboratory development.

Founded in 1988, Techshot is a technology development company that primarily serves customers working in microgravity. The company's FabLab effort is focused on producing a ground-based prototype with the ability to mature into a flight demonstration aboard the International Space Station (ISS) within three years. The Techshot FabLab will be compatible with the space station's EXPRESS Racks. Remotely- 
controlled operations from Earth will manufacture multi-material components, including metals, ceramics, plastics and electronics. A schematic of the Techshot FabLab is shown in Figure 12.

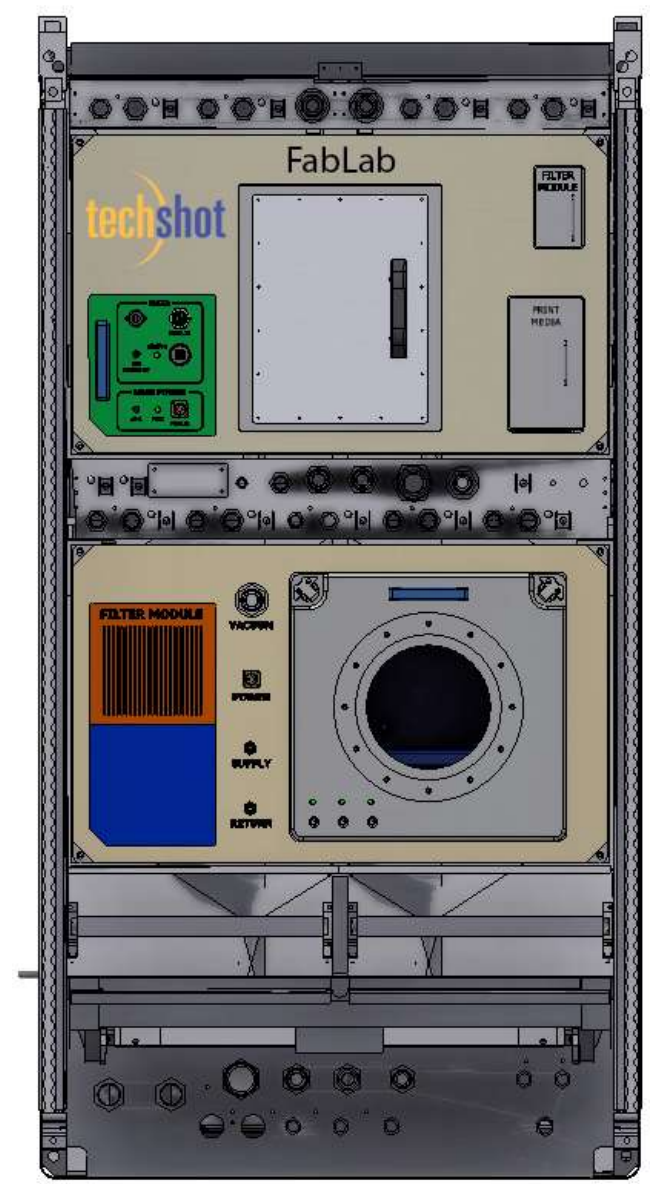

Figure 12. Techshot FabLab schematic. Image courtesy of Techshot.

The Tethers Unlimited, Inc. (TUI) Empyrean FabLab increases astronaut efficiency by providing autonomous processing and verification and validation services in a system designed for microgravity operation aboard the ISS. The Empyrean Fabrication Laboratory team, led by Tethers Unlimited Inc. (TUI) with IERUS Technologies, Inc. and BluHaptics, Inc., will develop the "Empyrean" FabLab to support NASA's long-duration and deep-space manned missions with capabilities for multi-material manufacturing and recycling. The Empyrean team will focus on a suite of support technologies for microgravity-enabled multi-material manufacturing, including robotic handling, quality control, autonomy, and teleoperation capabilities. These capabilities will enable sustainable in-space manufacturing in support of long-duration crewed missions, while minimizing demands upon astronaut time. 


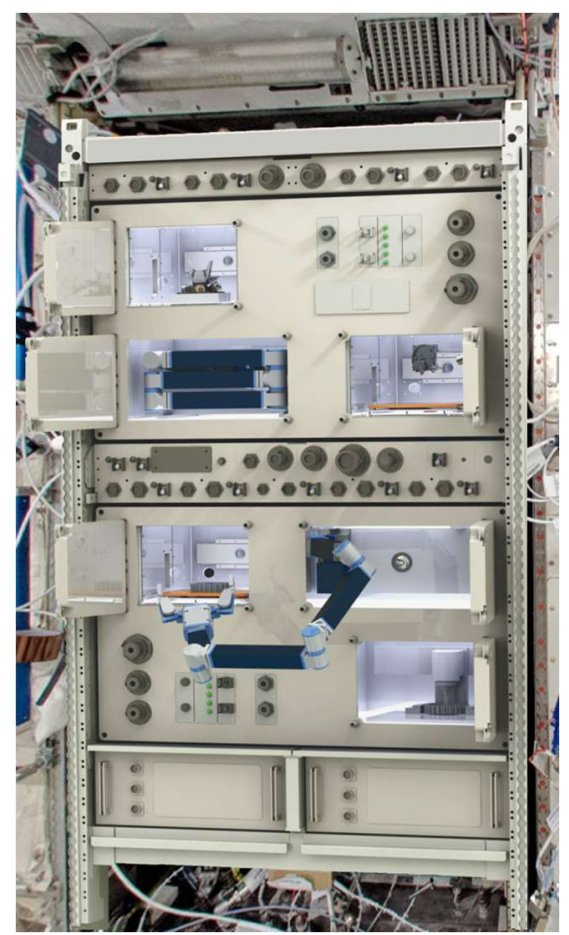

Figure 13. Schematic of Empyrean FabLab from Tethers Unlimited (TUI). Image courtesy of TUI.

Interlog Corporation (Interlog) will develop the Microgravity Multiple Materials Additive Manufacturing (M3AM) technology to provide on-demand manufacturing solutions for fabrication, maintenance, and repair on space missions. M3AM is capable of manufacturing various aerospace-grade metallic parts such as Aluminum, Titanium, Nickel, and other metallics. M3AM can also bond dissimilar materials (e.g., metals, glass epoxy, flexible ceramics). M3AM is enabled by Interlog's proprietary manufacturing technique that additively constructs a part via a focused bonding-energy mechanism. M3AM seeks to offer multi-material AM on a single platform, autonomous operation, dissimilar material bonding for electronics and PCB (Printed Circuit Board) fabrication as additional features, autonomous part removal, and multiple material feeding mechanisms.

\section{Conclusion}

In the coming years, NASA's in-space manufacturing project will continue maturation of manufacturing platforms and processes for use in a microgravity crewed environment. Key challenges to ISM implementation are socialization of capabilities with the design community and integration of ISM into exploration systems currently under development. The ISM team will continue to work with exploration system designers to identify high-value application areas and influence the design of these systems to take advantage of current and future ISM capabilities. ISM is a critical capability for the long endurance missions NASA seeks to undertake in the coming decades and also an important aspect of low earth orbit commercialization initiatives. To this end, ISM will continue to create and leverage partnerships with industry, small business, and academia to conduct technology development. The use of ISS as a proving ground for manufacturing systems will pave the way for the transition to earth-independent explorationclass missions in the post-ISS era. 


\section{References}

[1] S Owens, A., O. C. de Weck, W. Stromgren, W. Cirillo, and K. Goodliff. "Supportability Challenges, Metrics, and Key Decisions for Human Spaceflight." American Institute of Aeronautics and Astronautics SPACE Forum. Orlando, FL. 2017.

[2] Owens, A. and O. DeWeck. "Systems Analysis of In-Space Manufacturing Applications for International Space Station in Support of the Evolvable Mars Campaign." American Institute of Aeronautics and Astronautics SPACE Forum. Long Beach, CA. 2016.

[3] Prater, T.J., Q. Bean, N. Werkheiser, et al. "Summary Report on Results of the 3D Printing in Zero G Technology Demonstration Mission, Volume 1.” NASA/TP-2016-219101. NASA Technical Publication Series. July 2016.

[4] Prater, T.J., Q. Bean, N. Werkheiser, et al. "Analysis of specimens from phase I of the 3D Printing in Zero G Technology demonstration mission." Rapid Prototyping Journal 23 (2017): p. 1212-1225.

[5] Prater, T.J., N. Werkheiser, F. Ledbetter. "Complete Results from the 3D Printing in Zero-G Technology Demonstration Mission, Volume II." NASA Technical Publication Series (in queue, estimated date of publication March 2018).

[6] Additive Manufacturing Facility (AMF) User's Guide. Made in Space. 2016. http://madeinspace.us/wpcontent/uploads/AMF-User-Guide.pdf

[7]_ASTM C297. "Standard Test Method for Flatwise Tensile Strength of Sandwich Constructions." ASTM International. West Conshohocken, PA. 2016.

[8] O'Hara IV, W., J. Kish, and M. Werkheiser. "Turn-Key Use of an Onboard 3D Printer for ISS Operations." NASA Technical Report. 1 January 2017. www.ntrs.nasa.gov/search.jsp?R=20170003300\&qs=N\%3D4294933941

[9] Guthrie, Patricia. "Sporks in Space: Bothell Firm Brings recycling to final frontier." Herald Business Journal. 24 August 2016. www.heraldnet.com/business/sporks-in-space-bothell-firm-brings-recycling-to-final-frontier/

[10] ASTM D638. "Standard Test Method for Tensile Properties of Plastics," ASTM International. ASTM International. West Conshohocken, PA, 2014.

[11] Prater, T., N. Werkheiser, F. Ledbetter. “Toward a Multimaterial Fabrication Laboratory: In-Space Manufacturing as an Enabling Capability for Long Endurance Human Space Flight." American Institute of Aeronautics and Astronautics SPACE Forum. Orlando, FL. September 2017.

[12] Graff, K. "Ultrasonic Additive Manufacturing." ASM Handbook: Welding Fundamentals and Processes 6A (2011): pp. 731-741.

[13] Muhlbauer, Rachel. "Metal Advanced Manufacturing Bot Assembly (MAMBA) Process." Small Business Innovative Research (SBIR) abstract. 2017. http://sbir.nasa.gov/SBIR/abstracts/17/sbir/phase1/SBIR-17-1H7.02-9710.html

[14] Muhlbauer, Rachel. "Food-safe, skin contact-safe, and medical device 3D printing for manned space missions." Proceedings of the National Space and Missile Materials Symposium. June 2017.

[15] Kurk, Andy. "Sintered Inductive Metal Printer with Laser Enhancement." Proceedings of the National Space and Missile Materials Symposium. June 2017.

[16] "CRISSP Custom Recyclable International Space Station Packaging." Small Business Innovative Research (SBIR) abstract. 2017. www.sbir.gov/sbirsearch/detail/1148879

[17] Snyder, Ryan. "Reversible Thermoset Materials for in situ Resource Utilization." National Space and Missile Materials Symposium. June 2017.

[18] Vellinger, John. Software and Tools for Electronic Printing in Space. Small Business Innovative Research (SBIR) abstract. 2017. http://sbir.nasa.gov/SBIR/abstracts/17/sbir/phase1/SBIR-17-1-H7.01-9552.html 
[19] Renn, M., M. Schrandt, J. Renn, and J. Feng. "Localized Laser Sintering of Metal Nanoparticle Inks Printed with Aerosol Jet Technology for Flexible Electronics." Journal of Microelectronics and Electronic Packaging 14 (2017): pp. 132-139.

[20] "In-Space Manufacturing (ISM) Multimaterial Fabrication Laboratory (FabLab)." Broad Agency Announcement. 11 April 2017.

www.fbo.gov/index $? \mathrm{~s}=$ opportunity \&mode $=$ form $\&$ tab $=$ core $\& i d=8 \mathrm{a} 6 \mathrm{ebb} 526 \mathrm{~d} 8 \mathrm{bf} 8 \mathrm{fb} 9 \mathrm{c} 6361 \mathrm{cb} 8 \mathrm{~b} 50 \mathrm{c} 1 \mathrm{f} 8 \& \_\mathrm{cview}=1$

[21] Warner, Cheryl. "NASA Selects Three Companies to Develop 'FabLab' Prototypes." NASA Press Release. 7

December 2017. www.nasa.gov/press-release/nas-selects-three-companies-to-develop-fablab-prototypes 